J. Clin. Chem. Clin. Biochem.

Vol. 14, 1976, pp. 443-447

\title{
Bivariate Wertung von Befunden: Immunglobulin G und Albumin des Liquor cerebrospinalis
}

\author{
Von W. Bernhardt und B. Weisner \\ Neurologische Universitäts-Klinik und-Poliklinik (Direktor: Prof. Dr. Dr. R. Janzen) Hamburg-Eppendorf
}

(Eingegangen am 17. März/28. Maí 1976)

Zusammenfassung: Albumin und Immunglobulin $\mathrm{G}$ wurden in nativem, lumbal entnommenem Liquor cerebrospinalis mit der radialen Immundiffusion bestimmt. Bei Normalpersonen $(n=127)$ waren die Konzentrationen von Albumin und Immunglobulin $G$ annähernd normalverteilt; die Werte korrelierten linear miteinander $(r=0,60)$. Als bivariater Normalwertbereich wurde die Streuungsellipse berechnet, welche 95\% der Wertepaare umschließt. Diese klinische Grenze diskriminierte schärfer zwischen unauffälligen und pathologisch veränderten Wertepaaren als die beiden eindimensionalen Normalwertbereiche $\bar{x} \pm 2 \mathrm{~s}$. Der bivariate Normalwertbereich war daher von Vorteil für die Wertung der Befunde im einzelnen Krankheitsfall. Die zweidimensionale Betrachtung der Befunde in klinisch definierten Gruppen von Patienten ( $n=239)$ ergab ebenfalls zusätzliche Gesichtspunkte: Bei vielen Syndromen (bakteriellen Encephalomeningitiden, Polyneuropathien, myatrophen Lateralsklerosen) korrelierten die Albumin- und Immunglobulin G-Konzentrationen miteinander, vermutlich infolge einer Störung der Blut-Liquor-Schranke. Eine Sonderstellung nahmen Meningoencephalitis bei Virusinfektion und Encephalomyelitis disseminata im Sinne der Multiplen Sklerose ein: in diesen Gruppen stieg im Liquor die Immunglobulin G-Konzentration unabhängig von der Albumin-Konzentration.

\section{Bivariate evaluation of laboratory findings: immunoglobulin $G$ and albumin in cerebrospinal fluid}

Summary: Using radial immunodiffusion, albumin and immunoglobulin $G$ were determined in non-preconcentrated cerebrospinal fluid from 127 controls and from 239 patients. In controls the concentrations of albumin and immunoglobulin $G$ followed normal distribution. The two variables were correlated linearly $(r=0.60)$. The elliptic bivariate normal range was calculated, and was found to contain $95 \%$ of the paired values. As a clinical limit, this range discriminated more effectively between normal and altered pairs than the two one-dimensional normal ranges $\bar{x} \pm 2 s$, thus improving the evaluation of laboratory findings in the single case. Likewise in clinically defined groups of patients, bivariate evaluation of results provided additional evidence: In many distinct clinical syndromes, e. g. bacterial encephalomeningitis, polyneuropathy, amyotrophic lateral sclerosis, albumin and immunoglobulin $\mathrm{G}$ concentrations exhibited an especially close correlation, probably resulting from damage to the blood-cerebrospinal fluid barrier. Howevèr, no correlation of these two variables was detected in acute encephalomeningitis due to virus infection, and in multiple sclerosis: in these groups, immunoglobulin $\mathrm{G}$ concentrations were elevated independently of albumin. Since evidence is lacking as to the pathogenesis of multiple sclerosis, it seems noteworthy that the same phenomenon was observed in a well-defined group of viral infections.

\section{Einfuihrung}

Mit der Immundiffusion kann Immunglobulin G trotz seiner geringen Konzentration in nativem Liquor (10 bis $14 \mathrm{mg} / \mathrm{l}$ ) bestimmt werden (1). Seither dient die Methode zunehmend als fester Bestandteil der üblichen Liquordiagnostik. Sie zeichnet sich durch ihre Zuver: lässigkeit aus. Dạgegen genügen die Ërfahrungen in der Wertung der Befunde noch nicht. Die Aussagen über Normalwertbereiche stützen sich auf kleine Kollektive (2-9). Schon Gottesleben \& Bauer (3) haben darauf hingewiesen, daß die Immunglobulin G-Konzentrationen des Liquors nicht isoliert gewertet werden können, sondern nur zusammen mit ihrem Anteil am Gesamtprotein, der in dem Quotienten Proteinfraktion/LiquorGesamtprotein ausgedrückt wird.

Im Liquor von Normalpersonen korrelieren die Konzentrationen von Immunglobulin $G$ und Albumin. Der ellipsenförmige, bivariate Normalwertbereich wird errechnet und der Beurteilung von Befunden in zehn Gruppen von Patienten zugrunde gelegt. 


\section{Material und Methoden}

\section{Immunologische Methoden}

Albumin und Immunglobulin $G$ wurden in nativem Liquor mit der radialen Immundiffusion (1) bestimmt. Verwandt wurden Low-Concentration-Partigen Immund iffusionsplatten der Behringwerke Marburg. Der Variabilitätsquotient in der Serie betrug $5 \%$.

\section{Normalpersonen}

In der Gruppe der Normalpersonen $(n=127)$ war die Lumbalpunktion wegen eines begründeten Verdachtes auf eine neurologische Erkrankung als Ausschlußuntersuchung durchgeführt worden. Der Verdacht ließ sich nicht erhärten. In diesen Liquorproben betrug die Zellzahl bis 12/3, die Trübung in der Normomastixreaktion bis V und das Gesamteiweiß bis $520 \mathrm{mg} / \mathrm{l}$. Vor der Lumbalpunktion waren die Probanden nicht bettlägerig; sie haben sich nach Belieben bewegt.

\section{Patienten}

Liquorproben von 239 neurologischen Patienten wurden untersucht. Die Ergebnisse wurden nach klinischen Diagniosen gruppiert. In den Fällen mit akuten, entzündlichen Reaktionen (bakterielle oder virale Encephalomeningitis) war die Lumbalpunktion während der ersten Krankheitswoche durchgeführt worden. Diese Patienten waren bettlägerig. Die Patienten der anderen Krankheitsgruppen haben sich vor der Lumbalpunktion je nach Belieben und Kräften bewegt.

\section{Statistische Auswertung}

Die Verteilungsform der Meßwerte wurde in dem linearen und logarithmischen Wahrscheinlichkeitsnetz geprüft. Der Zusammenhang zwischen den beiden Merkmalen Albumin- und Immunglobulin G-Konzentration im Liquor wurde durch die Streuungsellipse dargestellt (10). Die Gleichung der Ellipse ist gegeben durch (11):

$u^{2}-2 r u v+v^{2}=A$

$A=2 \frac{n-1}{n-2} F_{(2, n-2)} \cdot\left(1-r^{2}\right)$

$u=\frac{x-\bar{x}}{s_{x}} ; v=\frac{y-\bar{y}}{s_{y}} ; F=F-$ Verteilung

Dabei ergibt sich der Kurvenparameter $\mathbf{A}$ aus dem Stichprobenumfang und dem Korrelationskoeffizienten nach Gleichung (II). In der Verteilung der Meßwerte (oder der transformierten Werte) darf kein Unterschied zur Normalverteilung nachweisbar sein.

Berechnet wurde jeweils die Streuungsellipse, welche 95\% der Wertepaare umschließt. Die Rechnung wurde ausgefuhrt mit dem
Großrechner Telefunken TR 440 des Rechenzentrums der Universität Hamburg. Wir danken Herrn Dipl.-Math. W. Rehpenning (Medizinische Dokumentation und Statistik des Fachbereichs Medizin Hamburg) für die Beratung und für das FORTRANProgramm.

\section{Ergebnisse}

Die Prüfung mit Hilfe von Wahrscheinlichkeitspapier ergibt, daß die Konzentrationen von Albumin und Immunglobulin $\mathbf{G}$ im Liquor bei Normalpersonen annähernd normalverteilt sind. Mittèlwert und Standardabweichung sind in Tabelle 1 enthalten.

In Abbildung 1 sind die Konzentrationen von Immunglobulin $\mathrm{G}$ in den Liquores von Normalpersonen in Ab-

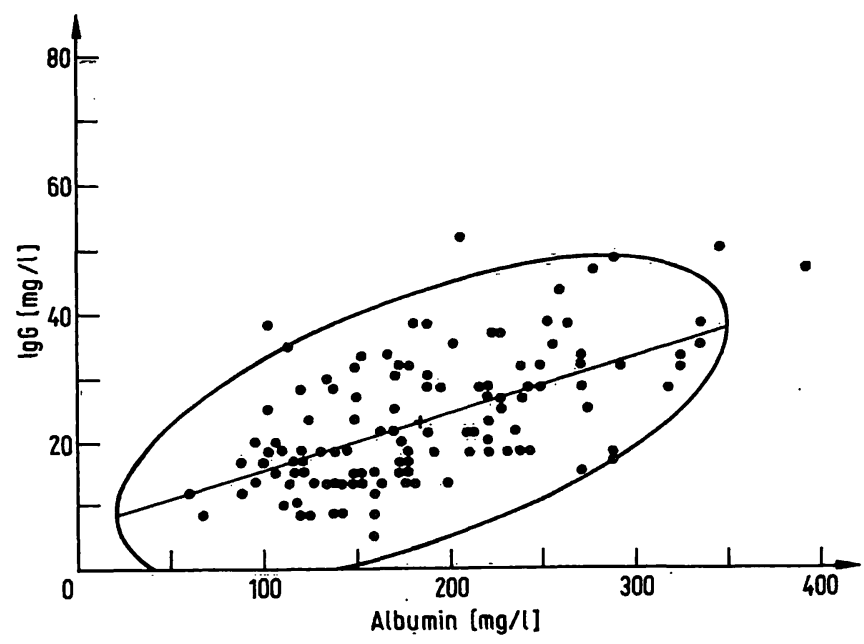

Abb. 1. Wertepaare der Albumin- und Immunglobulin G-Konzentrationen in lumbal entriommenen Liquores von Normalpersonen $(n=127)$. Eingetragen sind die Streuungsellipse (95\%-Toleranzbereich als bivariater Normalwertbereich) sowie die Regressionsgerade y (x). Der Mittelpunkt der Ellipse (+) ist zugleich das arithmetische Mittel der Albumin- und Immunglobulin G-Konzentriationen.

Tab. 1. Albumin- und Immunglobulin G-Konzentrationen in lumbal entnommenen Liquores von Normalpersonen und Patienten, gruppiert nach klinischen Diagnosen. Ergebnisse der Korrelations- und Regressionsanalyse. $n=$ Z̈ahl der Fälle, $\bar{x}, \bar{y}=$ arithmetisches Mittel der Gruppen; $s=$ Standardabweichung; $r=$ Korrelationskoeffizient $($ Pearson- $r) ; \alpha=$ Irrtumswahrscheinlichkeit; $b_{\mathbf{y} . \mathbf{x}}=$ Regressionskoeffizient für Schätzung von $\mathbf{y}$ an der Stelle $\mathbf{x} ; \mathbf{b}_{\mathbf{0}}=$ Achsenabschnitt auf der Ordinate.

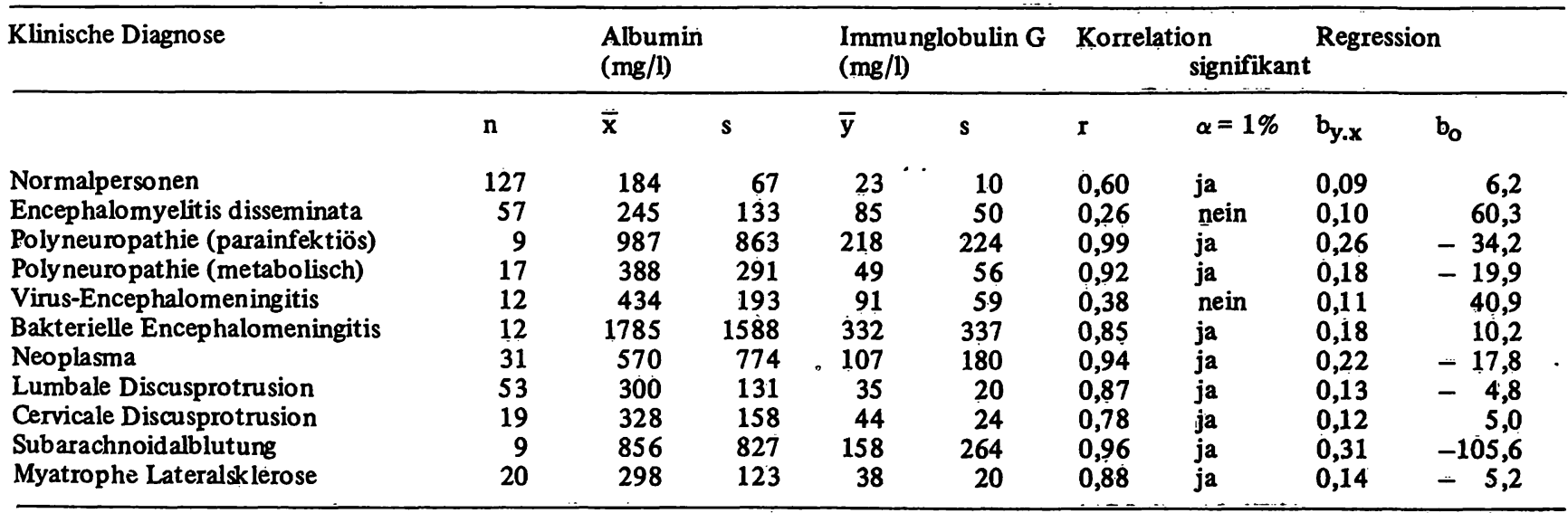


hängigkeit von der Albuminkonzentration dargestellt: der lineare Zusammenhang der Werte ist deutlich, der Korrelationskoeffizient r beträgt 0,60 (signifikant verschieden von Null auf dem 1\%-Niveau, Tab. 1). Eingetragen in die Abbildung 1 sind außerdem die Streuungsellipse, die den 95\%-Toleranzbereich umschließt, sowie die Regressionsgerade y (x). Der Kurvenparameter A der Ellipse (Gleichung I, siehe Methoden) beträgt 3,952 .

Durch Vergleich der Streuungsellipsen, ihrer Lage im Koordinatensystem und ihrer Form, sind Aussagen über die verschiedenen Gruppen von Patienten möglich. In den Gruppen der Abbildung 2 korrelieren die Merkmale Albumin- und Immunglobulin G-Konzent ration noch enger miteinander als in der Gruppe der Normalpersonen, kenntlich an der schmaleren und längeren Form der Streuungsellipsen und ebenfalls abzulesen an dem Korrelationskoeffizienten (Pearson-r) in Tabelle 1. Die Gruppen „lumbale Discusprotrusionen“ und ,myatrophe Lateralskerose "ähneln einander; die Streuungsellipsen unterscheiden sich in der Neigung kaum von dem Normalwertbereich aus Abbildung 1, und sie umgreifen nur ein etwa doppelt so großes Areal. Einen viel weiteren Bereich des Koordinatensystems umschließen die Streuungsellipsen der Gruppen „Neoplasma“ und „Polyneuropathie parainfektiös". Die schlanke Form der Ellipsen zeigt die strenge Korrelation der beiden Variablen bei diesen Kranken. In allen Gruppen kommen Wertepaare vor, die den Normalwertbereich nicht überschreiten.

Im Gegensatz zu den akuten Meningo-encephalitiden durch bakterielle Infektion ist jedoch bei den akuten entzündlichen Reaktionen durch Virusinfektion keine Korrelation der Konzentrationen von Immunglobulin G und Albumin zu beobachten. Dieser Zusammenhang fehlt ebenfalls in der Gruppe der Patienten mit Encephalo. myelitis disseminata (Tab. 1), deutlich auch in der fast kreisförmigen Streuungsellipse (Abb. 3). Dieses Kollektiv zeichnet sich gegenüber den Normalpersonen durch eine Verschiebung der Wertepaare in positiver Richtung der Ordinate aus.
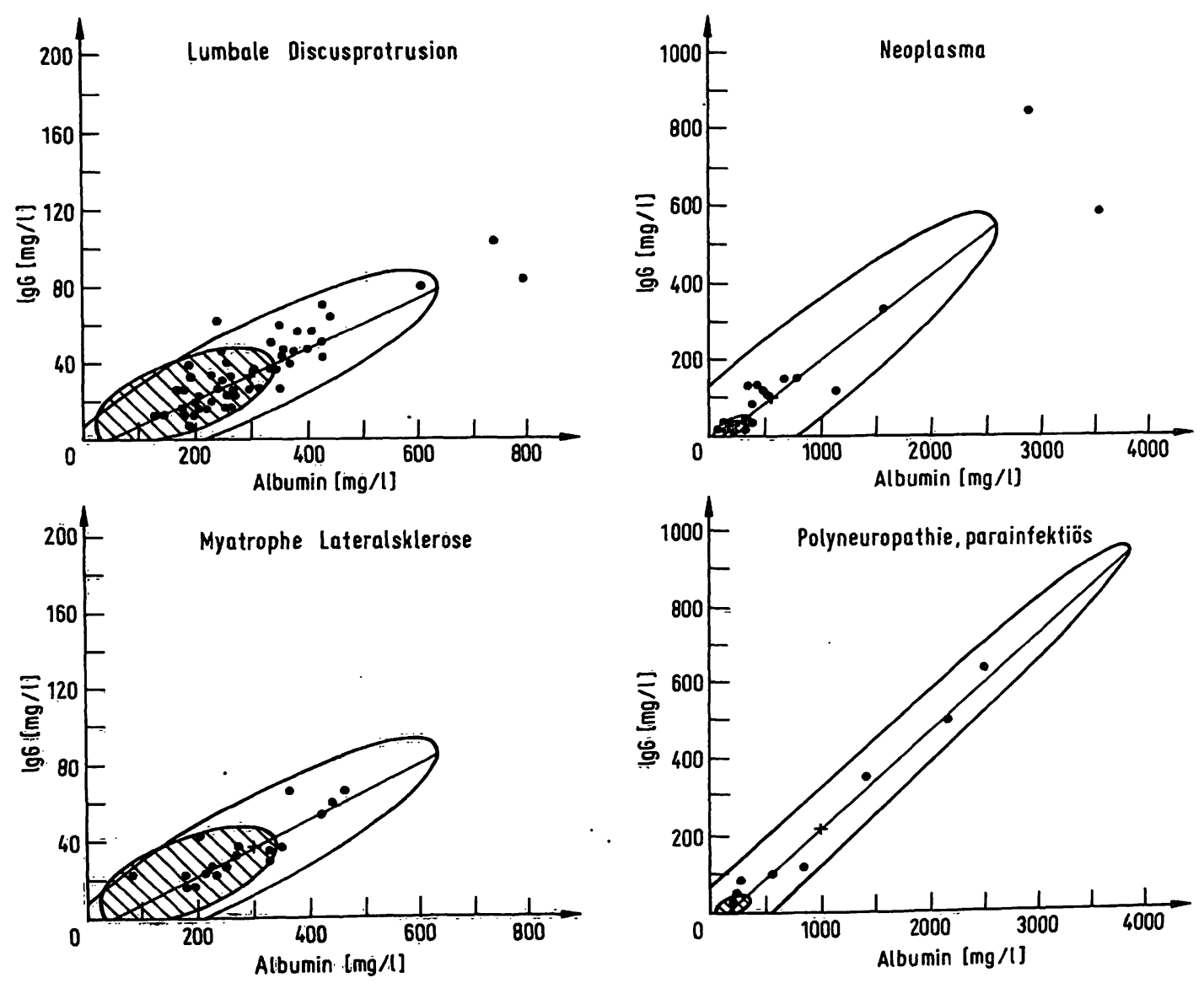

Abb. 2. Wertepaare der Albumin- und I mmunglobulin G-Konzentrationen in lumbal entnommenen Liquores von Patienten, geordnet nach klinischen Diagnosen. Dạs Verhältnis der Maßstäbe von Ordinate und Abszisse ist in allen vier Koordinatensystemen konstant. Eingetragen sind die Streuungsellipse des Kollektivs, die zugehörige Regressionsgerade $y(x)$ sowie der bivariate Normalwertbereich aus Abbildung 1 (schraffiert). Der Mittelpunkt der Ellipsen ( + ) ist zugleich das arithmetische Mittel der Albumin- und Immunglobulin G-Konzentrationen. 


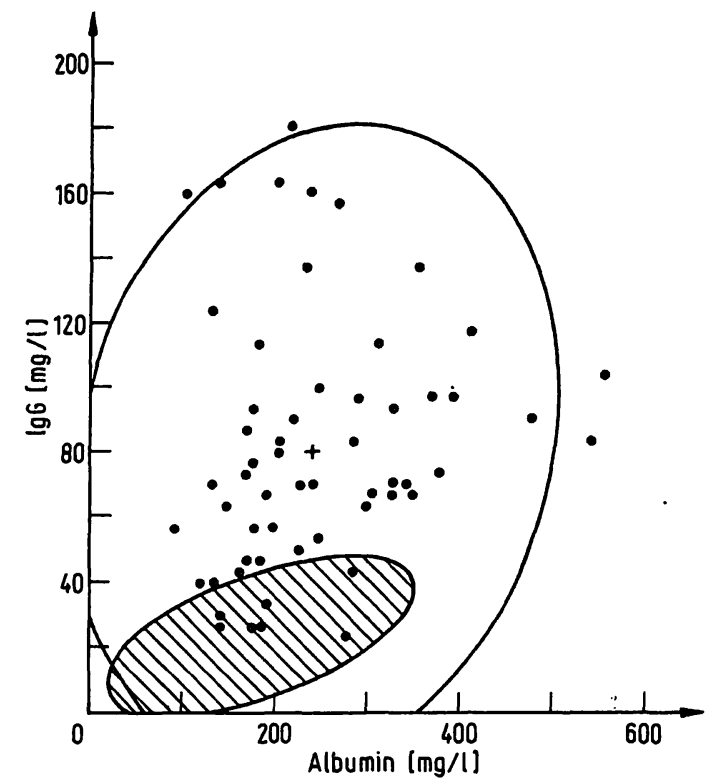

Abb. 3. Wertepaare der Albumin- und Immunglobulin G-Konzentrationen in lumbal entnommenen Liquores von Patienten mit Encephalomyelitis disseminata. Das Verhältnis der Maßstäbe von Ordinate zu Abszisse ist das gleiche wie in Abbildung 2. Eingetragen sind die (fast kreisförmige) Streuungsellipse sowie der bivariate Normalwertbereich aus Abbildung 1 (schraffiert). Der Mittelpunkt der Ellipse ( + ) ist zugleich das arithmetische Mittel der Albumin- und Immunglobulin G-Konzentrationen.

\section{Diskussion}

\section{Bivariater Normalwertbereich}

Grundlagen für die Berechnung eines bivariaten Normalwertbereiches der Albumin- und Immunglobulin G-Konzentration in lumbal entnommenem Liquor sind die Meßwerte in 127 Proben, die von „Normalpersonen“ stammen: Probanden, bei denen im Rahmen einer Ausschlußuntersuchung die übrigen klinischen und klinischchemischen Merkmale keine pathologischen Veränderungen erkennen ließen. In dieser Population sind beide Variablen normalverteilt, und sie korrelieren linear miteinander. Angesichts normalverteilter Kollektive werden üblicherweise $\bar{x} \pm 2 s$ als Schranken des Normalwertbereichs gewählt (12).

Anzugeben wäre also in diesem Fall je ein gesonderter Bereich für Albumin und für Immunglobulin G. Dadurch aber ginge für die Wertung der Befunde jene Information verloren, die in der Korrelation der beiden Variablen enthalten ist. Die Korrelation läßt sich übersichtlich mit der Streuungsellipse darstellen (10). Berechnet wurde der 95\%-Toleranzbereich (Abb. 1): Diese Ellipse umgrenzt einen bivariaten Normalwertbereich (Irrtumswahrscheinlichkeit $\alpha=0,05$ ). Nach Normierung der Variablen (siehe Methoden) läßt sich mit dem Kurvenparameter $\mathrm{A}=3,952$ auch rechnerisch prïfen, ob ein bestimmtes Wertepaar innerhalb des elliptischen Normalwertbereichs liegt.

Als ,klinische Grenze“ (13) diskriminiert dieser bivariate Normalwertbereich wesentlich schärfer als die beiden
Schranken $\bar{x} \pm 2 s$ (Tab. 1.): Wertepaare, deren Variablen (einzeln betrachtet) die Schranken $\bar{x} \pm 2$ s nicht überschreiten, werden trotzdem als pathologisch verändert gewertet, weil das Zusammentreffen einer AlbuminKonzentration $\bar{x}+2$ s gleichzeitig mit einer Immunglobulin G-Konzentration $\bar{y}-.2$ s auf Grund der Korrelation sehr unwahrscheinlich ist. Eine Erweiterung dieser Erwägungen führt zu dem Schluß, daß für andere klinische Fragestellungen manchmal multivariate Normalwertbereiche benötigt werden, wenn die vorhandene Information für die Wertung der Befunde ausgeschöpft werden soll.

\section{Wertung der Befunde}

Mit Hilfe der Streuungsellipse für Normalpersonen (Abb. 1) kann mit der vorgewählten Irrtumswahrscheinlichkeit $(\alpha=0,05)$ graphisch geprüft werden, ob ein bestimmtes Wertepaar den Toleranzbereich überschreitet. Die Anwendung dieses Verfahrens zeigt an den Beispielen der Abbildung 2, daß bei mehreren Wertepaaren der übliche Normalwertbereich $\bar{x} \pm 2 s$ (Tab. 1) zur Diskriminierung nicht ausgereicht hätte. Bedeutsam ist diese Feststellung insbesondere für die Gruppe ,myatrophe Lateralsklerose" angesichts der klinischen Regel, daß der Liquor dieser Patienten regelrecht zusammengesetzt sei. Die Berichte über pathologische Veränderungen häufen sich aber $(14,15,16)$.

Betrachtet man die Ergebnisse innerhalb klinisch definierter Gruppen von Patienten ebenfalls zweidimensional, so sind auf einen Blick Gruppenunterschiede zu erkennen. Alle Streuungsellipsen der Patientengruppen in Abbildung 2 zeichnen sich gegenüber der Ellipse der Normalpersonen (Abb. 1) durch eine schmalere und längere Form aus und zeigen auf diese Weise, daß die beiden Variablen enger miteinander korrelieren. Je ausgedehnter der Bereich wird, den eine Streuungsellipse im Koordinatensystem umgreift, desto steiler wird die Neigung der Achse der Ellipse (Abb. 2, Gruppe „Neoplasma“ und „Polyneuropathien parainfektiös"). Das bedeutet, der Anteil an Immunglobulin $G$ hat gegenüber dem Albumin zugenommen. Er nähert sich dem Verhältnis, wie man es im Serum findet $(17,18)$. Eine Korrelation zwischen den Konzentrationen dieser Proteinfraktionen in Serum und Liquor wird aber auch in extremen Fällen nicht nachweísbar (18). Trotzdem liegt der Verdacht nahe, daß den Veränderungen der Liquorzusammensetzung in diesen Gruppen eine Störung der sogenannten Blut-Liquor-Schranke zugrunde liege (19-24).

Die Abbildung 3 zeigt das Beispiel einer Gruppe von Patienten, in der keine Korrelation der beiden Merkmale Albumin- und Immunglobulin G-Konzentration im Liquor nachweisbar ist: die Streuungsellipse hesitzt eine fast kreisförmige Gestalt. Bei diesen Patienten mit Encephalomyelitis disseminata im Sinne der multiplen Sklerose ist die Immunglobulin G-Konzentration unab- 
hängig von der Albumin-Konzentration angestiegen. Das gleiche gilt für die Gruppe mit Virus-Encephalitis: die Regressionsgerade schneidet die Ordinate weit im positiven Bereich, ihre Steigung aber ist gegenüber den Normalpersonen kaum verändert (Tab. 1: $b_{0}$ und

\section{Literatur}

1. Augener, W. (1965), in: Proc. XII Coll. Protides of the Biological Fluids (Peeters, H. ed), Elsevier Amsterdam, S. 363-371.

2. Hartley, T. F., Merrill, A. \& Calman, N. (1966), Arch. Neurol. 15, 472-479.

3. Gottesleben, H. \& Bauer, H. (1967), Germ. Med. Mth. 12, $331-334$.

4. Tourtellotte, W. (1967), Trans. Am. Neurol. Assoc. 92 , 288-290.

5. Delank, H. W. (1971), Nervenarzt 42, 483-490.

6. Delpech, B. \& Lichtblau, E. (1972), Clin. Chim. Acta 37, 15-23.

7. Glasner, H. \& Wenig, Ch. (1973), Klin. Wochenschr. 51, 806-807.

8. Savory, J. \& Heintges, M. G. (1973), J. Neurol. 23, 953-958.

9. Weisner, B., Schnedler, R. \& Bernhardt, W. (1975), Nervenarzt 46, 532-538.

10. Lind ner, A. (1964), Statistische Methoden, 4. Aufl. S. 173 bis 185 , Birkhäuser, Basel.

11. Hoyer, G. (1972), Meth. Inform. Med. 11, 37-48.

12. Büttner, H. (1973), in: Optimierung der Diagno stik (Lang, H. Rick, W., Róka, L., Hrsg.) S. 233-241, Springer, Berlin, Heidelberg, New York. $\left.b_{y . x}\right)$. Angesichts der Frage, ob die Gruppe „Encephalomyelitis disseminata " uberhaupt als homogen anzusehen sei, kommt der Beobachtung desselben Phänomens in einer definierten Gruppe (Virus-Encephalitis) Bedeutung zu.

13. Elveback, L. R., Guillier, C. L. \& Keating, F. R. (1970), J. Amer. Med. Ass. 211, 69-75.

14. Gerstenbrand, F., Gründig, E., Scheidl, R. Siamany, M. \& Teufelmayer, R. (1971), Nervenarzt 42, 93-97.

15. Berlet, H. H. \& Pilz, H. (1972), Z. Neurol. 201, 310-325.

16. Müller-Jensen, A. \& Bernhardt, W. (1973), Nervenarzt 44, 143-149.

17. Schultze, H. E. \& Schwick, G. (1959), Clin. Chim. Acta 4, 15-25.

18. Weisner, B. \& Bernhardt, W. (1975), diese Z. 13, 245.

19. Frick, E. \& Scheid-Seydel, L. (1958), Klin. Wochenschr. 36, 66-69.

20. Frick, E. \& Scheid-Seydel, L. (1958), Klin. Wochenschr. 36, 857-863.

21. Delpech, B. \& Lichtblau, E. (1972), Clin. Chim. Acta 37, 15-23.

22. Link, H., Zettervall, O. \& Blennow, G. (1972), Z. Neurol. 203, 119-132.

23. Ganrot, K. \& Laurell, C. B. (1974), Clin. Chem. 20, $571-573$.

24. Felgenhauer, K. (1974), Klin. Wochenschr. S2, 1158 bis 1164 .
Priv.-Doz. Dr. W. Bernhardt

Neurologische Universitätsklinik und Poliklinik Martinistraße 52

D-2000 Hamburg 20 
